\title{
Das Gleiche aber nicht dasselbe - neue epidemiologische Einschätzung durch moderne molekularbiologische Methoden
}

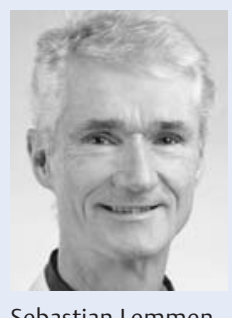

Sebastian Lemmen

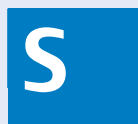

chnell unterstellt man eine nosokomiale Übertragung, wenn bei zwei oder gar mehreren Patienten Bakterien derselben Spezies, wie z. B. S. aureus oder E. coli aus mikrobiologischen Materialien oder in Screeninguntersuchungen nachgewiesen werden. Wenn es sich hierbei dann noch um Bakterien mit einem auffälligen Resistenzmuster wie beispielsweise MRSA, VRE oder 3-/4-MRGN oder auch um Clostridium difficile handelt, scheint eine Transmission schon fast bewiesen zu sein - was sonst?

Als Quelle hierfür wird üblicherweise der Indexpatient oder die unbelebte Umgebung - meist sein Patientenzimmer - und als Vektor die Hände des Personals oder kontaminierte Gegenstände (z. B. Stethoskop) angeschuldigt. Kommt es dann im weiteren stationären Verlauf bei mindestens zwei dieser Patienten zu einer Infektion, muss dies als Ausbruch gemeldet werdenungeachtet einer Bezugsgröße wie beispielsweise Größe der Station oder Anzahl der behandelten Patienten, um eine Rate berechnen zu können. In Zeiten eines investigativen Journalismus mit gelegentlichem Missionscharakter kann man dann sehr schnell den Hygieneskandal seines eigenen Krankenhauses in der Lokalpresse wiederfinden.

Patienten sind inzwischen jedoch zunehmend bereits bei der stationären Aufnahme mit diesen resistenten Bakterien auf der Haut oder im Darm kolonisiert. Aktuelle Daten des Nationalen Referenzzentrums für Surveillance (NRZ) zeigen, dass inzwischen MRSA zu ca. $90 \%$, VRE zu ca. $70 \%$, 3-MRGN zu ca. $80 \%$ und selbst 4-MRGN zu ca. 65\% mitgebracht wurden, diese Erreger sind also längst keine Krankenhauskeime mehr unabhängig davon, ob es sich um Patienten auf einer Intensiv- oder peripheren Station handelt (www.nrzhygiene.de).

Die wichtigsten Ursachen hierfür sind bestimmt eine Persistenz des Erregers nach früherer stationärer Akquisition, eine hohe Antibiotika-Anwendungsrate in der ambulanten Medizin und in der Tiermast sowie eine Transmission im häuslichen Bereich.
Vor diesem Hintergrund muss die Übertragbarkeit der Ergebnisse eines kürzlich erschienenen systematischen Reviews mit Metaanalyse kritisch hinterfragt werden [1]:

Hier konnte gezeigt werden, dass das Risiko mit einem multiresistenten Erreger oder C. difficile im Krankenhaus kolonisiert, bzw. infiziert zu werden, doppelt so hoch bei MRSA und VRE und fast 3-mal so hoch bei MRGN ist, wenn man in demselben Patientenzimmer untergebracht wird, in dem zuvor ein Patient mit dem gleichen MRE lag. Ein solches Ergebnis hat natürlich gravierende Auswirkungen auf die Bedeutung der unbelebten Umgebung auf die Transmission im Krankenhaus. Intensivierte Reinigungs- und Desinfektionsmaßnahmen wären die Folge, die bereits bestehende Angst in der Bevölkerung vor Krankenhauskeimen würde sicher noch mehr zunehmen.

Diese Metaanalyse basiert auf 7 retrospektiven Einzelarbeiten, bei welchen - mit einer einzigen Ausnahme keine molekularbiologische Typisierung durchgeführt wurde [1]. Die einzige Studie mit molekularbiologischer Typisierung zeigte, dass die bakterielle Umgebung keine signifikante Bedeutung bei der Übertragung von in diesem Fall ESBL-bildenden gramnegativen Erregern hatte [2]. Inwiefern diese Arbeit dann in die Metaanalyse eingeflossen ist, bleibt unklar.

Selbstverständlich kommt es zu Erregerübertragungen - kein Neugeborenes verlässt steril das Krankenhaus - und gelegentlich ergibt sich hieraus auch eine Häufung, die selten auch zu einer Ausbruchssituation führen kann. Stehen diese Häufungen jedoch nicht in einem eindeutigem epidemiologischen Zusammenhang, kann erst eine molekularbiologische Identifizierung des Genotyps die Übertragung bestätigen, ein identischer Phänotyp mit Differenzierung der Erreger auf Speziesebene, selbst mit identischen Antibiogramm, ist hier nicht ausreichend. 
In dieser Ausgabe von „Krankenhaushygiene up2date“ wird eine sehr gute und aktuelle Übersicht über die äußerst komplexe Infektionsentität verursacht durch C. difficile gegeben. Auch hier wird durch aktuelle epidemiologische Arbeiten unter Verwendung diskriminierender molekularbiologischer Methoden ein anderes Bild bzgl. der Übertragung von C. difficile gezeichnet: Eine Transmission im Krankenhaus scheint ein deutlich selteneres Ereignis zu sein, als bisher angenommen. Durch Sequenzierung des gesamten Genoms konnte erstmals 2013 in einer Grafschaft in England eine vergleichende Epidemiologie für C. difficile Infektionen beschrieben werden [3]. Unter fast 1000 Patienten wurde bei $65 \%$ überhaupt keine genotypische Identität der Stämme nachgewiesen, nur bei ca. 20\% der Patienten bestand vorher überhaupt ein Krankenhausaufenthalt - als Reservoire werden hier z. B. die unbelebte Umgebung, Tiere oder Nahrung diskutiert. Bei nur wenigen Patienten ist eine Übertragung im Krankenhaus aufgrund eines zeitgleichen Aufenthaltes auf derselben Station möglich.

Im Rahmen einer prospektiven Beobachtungsstudie zwischen 2003 und 2013 wurde am Basler Kantonsspital die nosokomiale Transmissionsrate von 845 Patienten mit C. difficile-assoziierter Diarrhö (CDAD) untersucht [4]. In der Schweiz ist es nicht üblich, Patienten mit CDAD zu isolieren und so konnten 472 Kontaktpatienten mit einem rektalen Abstrich auf C. difficile untersucht werden. In 6,6\% (31/472 Patienten) konnte $\mathrm{C}$. difficile nachgewiesen werden, aber nur in $1,3 \%$ (6/472 Kontaktpatienten) wurde der identische Stamm in der Ribotypisierung nachgewiesen. Nach Sequenzierung des gesamten Genoms (next generation sequencing) von 4 dieser 6 Stämme zeigte sich nur in zwei Stämmen eine identische Klonalität. Auch hier scheint also die Transmission im Krankenhaus ein äußerst seltenes Ereignis, die Hypothese einer endogenen Reaktivierung, getriggert durch Antibiotika und/ oder Protonenpumpen-Inhibitoren, scheint wahrscheinlicher.
Zusammenfassend ermöglichen moderne, gut diskriminierende molekularbiologische Methoden die Unterscheidung zwischen Transmission versus zufällige Häufung, durch Nachweis desselben Genotyps. Insbesondere bei multiresistenten Erregern überwiegt aktuell in Deutschland der Eintrag aus der Ambulanz in die Krankenhäuser bei weitem eine Erstdetektion im Krankenhaus, sodass hier - insbesondere wenn es sich nicht um einen offensichtlichen epidemiologischen Zusammenhang handelt - von einer Transmission erst bei nachgewiesenem identischem Genotyp gesprochen werden sollte.

Die aktuellen molekularbiologischen Studien bei C. difficile zeigen, dass es wohl außerhalb der Krankenhauses ein Vielfalt unterschiedlichste Reservoire für die Sporen von C. difficile gibt, die wohl deutlich häufiger zu einer Kolonisation/Infektion führen als die bisher in Deutschland angenommene Transmission im Krankenhaus.

\section{Literatur}

1 Mitchell BG, Dancer SJ, Anderson M et al. Risk of organism acquisition from prior room occupants: a systematic review and meta-analysis. Journal of Hospital Infection 2015; 91 : $211-217$

2 Ajao AO, Johnson JK, Harris AD et al. Risk of acquiring extended-spectrum ß-lactamase-producing Klebsiella Species and Escherichia coli from prior room occupants in the intensive care unit. Infect Control Hosp Epidemiol 2013; 34: 453-458

3 Eyre DW, Cule ML, Wilson DJ et al. Diverse sources of $C$. difficile infection identified of whole-genome sequencing. $N$ Engl ] Med 2013; 369: 1195-1205

4 Widmer A, Frei R, Lawley T et al. Dealing with Clostridium difficile - Transmissibility of $C$. difficile without contact isolation: results from a prospective observational study. Kopenhagen: University Hospital Basel-Switzerland; 2015: Poster O267, ECCMID, 